\title{
Maternal embryonic leucine zipper kinase inhibits epithelial-mesenchymal transition by regulating transforming growth factor- $\beta$ signaling
}

\author{
JIANJIAN CHENG, BINYU QIN, BAO LIU, TAIBO HUANG, YUGUANG LI and LIJUN MA \\ Department of Respiration and Critical Care and Emergency Medicine, \\ Henan Provincial People's Hospital, Zhengzhou, Henan 450003, P.R. China
}

Received January 30, 2016; Accepted February 27, 2017

DOI: $10.3892 / \mathrm{ol} .2017 .6081$

\begin{abstract}
Maternal embryonic leucine zipper kinase (MELK) performs an important role in self-renewal and proliferation of progenitor cells or tumor stem cells, and is expressed in aggressive cancers, contributing to tumorigenesis. However, the function of MELK in metastasis is unknown. In the present study, the lung cancer A549 cell line was utilized in order to study the role of MELK in epithelial-mesenchymal transitions (EMTs), the initial step of tumor metastasis. It was identified that transforming growth factor- $\beta$ (TGF- $\beta$ ) could downregulate the expression of MELK, and that MELK could inhibit EMT by regulating TGF- $\beta$ signaling. MELK can interact with Smad proteins, which represses TGF- $\beta /$ Smad-mediated signaling activity. The findings of the present study identified the effect of MELK in TGF- $\beta$ signaling and the EMT process.
\end{abstract}

\section{Introduction}

Maternal embryonic leucine zipper kinase (MELK) is a member of the adenosine monophosphate-activated protein kinase family of kinases. MELK was first cloned from the mouse egg and preimplantation embryo (1), and is expressed in progenitor cells and tumor stem cells of aggressive cancers, including glioblastomas and breast cancers (2-4). MELK promotes proliferation by phosphorylating cell division cycle $25 \mathrm{~B}$ and apoptosis signal-regulating kinase $1(5,6)$ and MELK induces apoptosis through phosphorylation of p53 at the Ser15 site (7). In a number of studies, the anti-apoptosis function of MELK was demonstrated through its regulation of p53 expression or interaction with B cell lymphoma-G $(8,9)$.

Correspondence to: Professor Lijun Ma, Department of Respiration and Critical Care and Emergency Medicine, Henan Provincial People's Hospital, 7 Weiwu Road, Zhengzhou, Henan 450003, P.R. China

E-mail: malijun1891@126.com

Key words: maternal embryonic leucine zipper kinase, epithelialmesenchymal transition, transforming growth factor- $\beta$, Smad, A549
Numerous studies have indicated that MELK is associated with tumor prognosis; MELK expression is increased in clinical samples of breast cancer or high-grade prostate cancer $(10,11)$. At present, numerous studies on MELK have focused on the tumorigenesis stage, and to the best of our knowledge, there has been no study investigating the function of MELK in tumor metastasis.

Tumor metastasis is a complex process, involving a single tumor cell disassociating from the tumor mass, migrating in the extracellular matrix, intravasating into the blood stream, extravasating from the blood vessel and colonizing distant organs (12). Epithelial-mesenchymal transition (EMT) is an important step for the initiation of tumor metastasis. When EMT occurs, epithelial tumor cells transition to mesenchymal-like cells, lose the expression of intercellular E-cadherin and acquire the expression of $\mathrm{N}$-cadherin and $\alpha$-smooth muscle actin, allowing tumor cells to migrate and enter the blood stream (13). A number of factors regulate the EMT. Among these, transforming growth factor- $\beta$ (TGF- $\beta$ ) signaling is intensively studied. TGF- $\beta$ signaling can upregulate the expression of transcription factors including Snail, ZEB and Twist, which repress the expression of E-cadherin and induce the expression of N-cadherin and fibronectin (13).

In the present study, the lung cancer A549 cell is utilized as an EMT model to observe the role of MELK in metastasis. Knockdown of MELK was identified to promote EMT by upregulation of TGF- $\beta$ signaling.

\section{Materials and methods}

Cell culture and plasmids. The A549 cell line was bought from American Type Culture Collection (Manassas, VA, USA), and was cultured in Dulbecco's modified Eagle's medium/low glucose medium containing $10 \%$ fetal bovine serum (HyClone; GE Healthcare Life Sciences, Logan, UT, USA), $2 \mathrm{mM}$ glutamine, penicillin and streptomycin (Thermo Fisher Scientific, Inc., Waltham, MA, USA). A549 cells were treated with TGF- $\beta 1$ (R\&D Systems, Inc., Minneapolis, MN, USA) at a concentration of $5 \mathrm{ng} / \mathrm{ml}$. The plasmid p3TP reporter gene, hemagglutinin (HA)-Smad2, HA-Smad3 and HA-Smad4 were stored by the central laboratory of Henan Provincial People's Hospital (Zhengzhou, China). The plasmid 
A

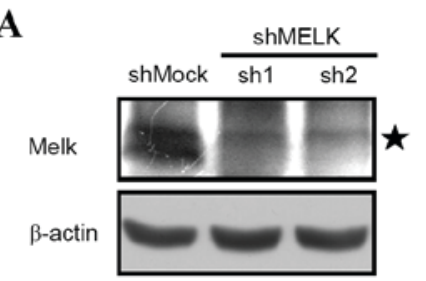

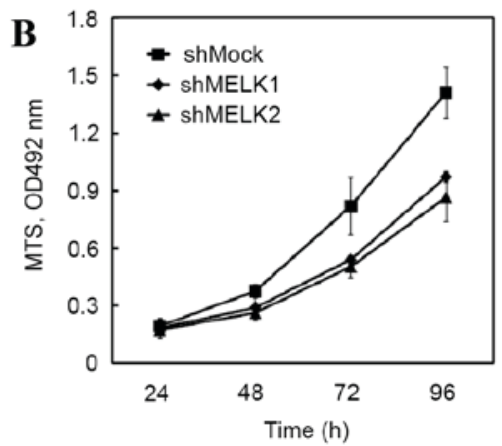

C

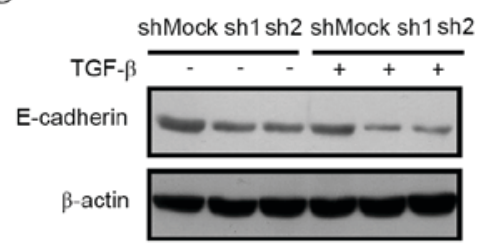

D
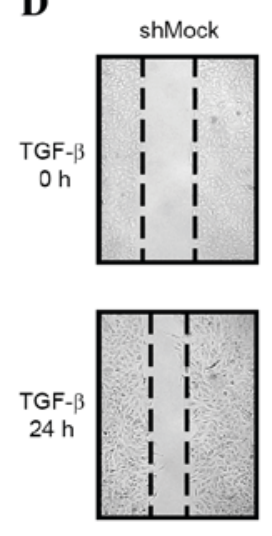

ShMELK1
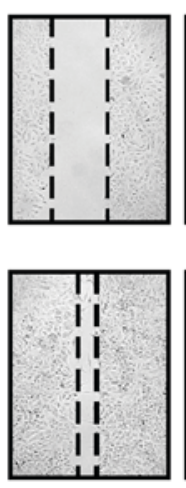

ShMELK2
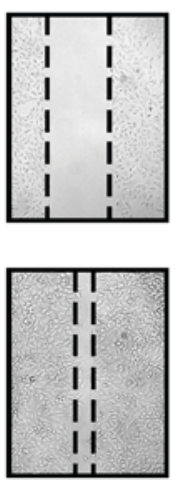
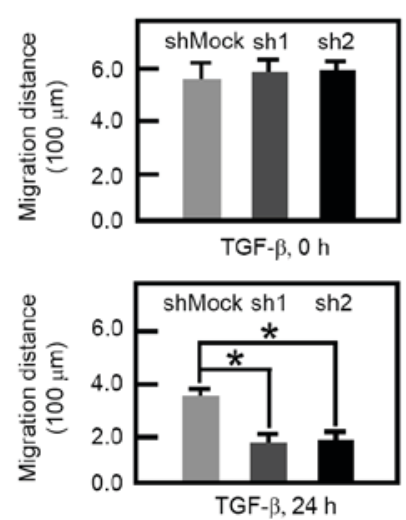

Figure 1. Knockdown of MELK promotes the EMT. (A) MELK expression was detected by western blot analysis, showing that MELK was decreased in shMELK (sh1 and sh2) cells compared with that in shMock cells. The star symbol shows the MELK band. (B) Proliferation rate was measured using MTS solution. Proliferation was decreased in shMELK1 and shMELK2 cells compared with shMock cells. (C) E-cadherin level was detected prior to or following TGF- $\beta$ stimulation. Western blot analysis shows that E-cadherin was dramatically decreased in shMELK (sh1 and sh2) cells. (D) Wound healing assay. Confluent A549 cells were scratched and then stimulated with or without TGF- $\beta$ for 24 h. Images were captured at 0 or 24 h, showing that shMELK (sh1 and sh2) cells migrate faster than shMock cells following TGF- $\beta$ stimulation. The quantification of distance is presented. The experiments were performed in triplicate. ${ }^{*} \mathrm{P}<0.05$. TGF- $\beta$, transforming growth factor- $\beta$; MELK, murine embryonic leucine zipper kinase; sh, short hairpin.

Flag-MELK was constructed through polymerase chain reaction (PCR) amplification (upstream primer, 3'-CGGGATCCA TGAAAGATTATGATGAACTTC-5' and downstream primer, 3'-CCCTCGAGTACCTTGCAGCTAGATAGGATG-5'), and ligation to plasmid pCS4-3Flag.

RNAi interference. The sequences (sh1, AACCCAAGGGTA ACAAGGATT and sh2, CAGGCAAACAATGGAGGAT) for RNAi interference are as previously described (14). The synthesized oligonucleotides were annealed and constructed into the pLKO.1 plasmid (Addgene, Inc., Cambridge, MA, USA) (15). Following sequencing, the lentivirus was packaged in 293T cells using package plasmids psPAX2 and PMD2 G (Addgene, Inc.). The stable cell lines were built with shMock (sequence, 5'-CCTAAGGTTAAGTCGCCCTCG-3'), shMELK1 and shMELK2 lentiviruses.

Western blot analysis. The cells were harvested in radioimmunoprecipitation assay buffer, supplemented with proteinase cocktail (Roche Diagnostics, Basel, Switzerland), $50 \mathrm{mM}$ sodium orthovanadate and $10 \mathrm{mM}$ sodium fluoride. The protein concentration was determined using the bicinchoninic acid kit (Thermo Fisher Scientific, Inc.). The antibodies for western blot analysis were MELK (dilution, 1:200; cat. no. sc-48035; Santa Cruz Biotechnology, Inc., Dallas, TX, USA, ), E-cadherin (dilution, 1:400; cat. no. sc-7870; Santa Cruz Biotechnology, Inc.), p-Smad2 (dilution, 1:1,000; cat. no. 3108;
Cell Signaling Technology, Inc., Danvers, MA, USA), Smad2 (dilution, 1:1,000; cat. no. 3122; Cell Signaling Technology, Inc.) and b-Actin (dilution, 1:2,000; cat. no. sc-7210; Santa Cruz Biotechnology, Inc.).

Reverse transcription-polymerase chain reaction (RT-PCR). The A549 cells were stimulated with TGF- $\beta 1(5 \mathrm{ng} / \mathrm{ml})$ for different periods of time (1, 3, 6, 12 and $24 \mathrm{~h})$, and the cells were lysed in TRIzol (Thermo Fisher Scientific, Inc.). The RNA was extracted following the protocol for TRIzol (16). Following RNA quantification, cDNA was obtained by reverse-transcription using RT master mix with the gDNA remover kit (cat. no. FSQ-301; Toyobo Co., Ltd., Osaka, Japan). PCR was performed using the thunderbird SYBR qPCR mix kit (cat. no. QPS-201; Toyobo Co., Ltd.) on the iCycler (Bio-Rad Laboratories, Inc., Hercules, CA, USA) instrument. The $2^{-\Delta \Delta \mathrm{Cq}}$ method was used to analyze the data (17).

Proliferation assay. The shMock, shMELK1, and shMELK2 cells were seeded onto 96 -well plates. A total of $2 \times 10^{3}$ cells were used in each well. At 24, 48, 72 and $96 \mathrm{~h}$, the absorbance at $492 \mathrm{~nm}$ was read using a microplate reader (Elx808; Norgen Biotek Corp., Thorold, ON, Canada) for the quantification of cell numbers.

Wound healing assay. The shMock and shMELK cells were counted using a hemocytometer plate under a microscope 
(DMi1; Leica Microsystems GmbH, Wetzlar, Germany) using x20 magnification. A total of $3 \times 10^{5}$ cells were seeded onto a 6-well plate. Following overnight cell culture at $37^{\circ} \mathrm{C}$, the cells reached confluency. The $200 \mu \mathrm{l}$ pipette tips were used to scratch the cell layer. Following scratching, images were captured at $0 \mathrm{~h}$ immediately, and TGF- $\beta 1(5 \mathrm{ng} / \mathrm{ml})$ was added to the medium. After $24 \mathrm{~h}$, the cell scratch was observed, and the images were captured as shown in Fig. 1D. The distance of the scratch in the images was measured using Photoshop software (Adobe, San Jose, CA, USA). The experiments were performed in triplicate wells.

Reporter gene assay. The p3TP reporter gene was used to evaluate TGF- $\beta$ signaling activity. Briefly, $8 \times 10^{4}$ cells were seeded onto a 12 -well plate. In total, $1 \mu \mathrm{g}$ plasmids were transfected into each well using Lipofectamine ${ }^{\circledR} 2000$ (Thermo Fisher Scientific, Inc.). After $24 \mathrm{~h}$, transfected cells were treated with TGF- $\beta 1(5 \mathrm{ng} / \mathrm{ml})$ for another $24 \mathrm{~h}$. The reporter gene activity was measured using the dual-luciferase reporter assay system (Promega Corporation, Madison, WI, USA). Triplicate wells for each plasmid combination were performed.

Immunoprecipitation. For immunoprecipitation, HEK293 cells were seeded onto $60 \mathrm{~mm}$ dishes at a density of $8 \times 10^{5}$ cells per dish. Following overnight incubation at $37^{\circ} \mathrm{C}$, the plasmid combinations ( $4 \mu \mathrm{g}$ per dish) were transfected. After three days, the cells were harvested in Nonidet-P40 buffer (0.1\% NP40). A total of $300 \mu \mathrm{g}$ of cell lysate was used for the immunoprecipitation assay. In each tube, $2 \mu \mathrm{g}$ of HA antibody (dilution, 1:40; cat. no. sc-7392; Santa Cruz Biotechnology, Inc.) was added. Following overnight incubation on an agitator in a cold room, protein A/G beads (cat. no. sc-2003; Santa Cruz Biotechnology, Inc.) were added to pull down the immuno-complex. The pellets were washed with washing buffer $(0.9 \% \mathrm{NaCl}$ including $0.01 \% \mathrm{NP} 40)$ three times, and then loaded onto SDS-PAGE gel. Flag antibodies (dilution, 1:100; cat. no. F3165; Sigma-Aldrich; Merck Millipore, Darmstadt, Germany) were used to detect signals.

Statistical analysis. The reporter gene assay was repeated in triplicate. The statistical software SPSS v19.0 was used (IBM, Armonk, NY, USA). Student's t-test was used to analyze differences between groups. $\mathrm{P}<0.05$ was considered to indicate a statistically significant difference.

\section{Results}

MELK is downregulated by TGF- $\beta$ at the protein and $m R N A$ level. In order to study the role of MELK in EMT, a A549 lung cancer cell model was employed, which can be induced to transition between epithelial cells and mesenchymal-like cells, by TGF- $\beta$ stimulation. Numerous effectors, including interferon regulatory factor-1 and thyroid transcription factor-1, are involved in this process $(18,19)$. Following TGF- $\beta$ stimulation of A549 cells for $24 \mathrm{~h}$, it was identified that TGF- $\beta$ induces the phosphorylation of Smad2, and the expression of E-cadherin (a marker of EMT) is decreased (Fig. 2A). The present study identified that MELK expression is downregulated in A549 cells following TGF- $\beta$ stimulation (Fig. 2A). Similarly, the mRNA level of MELK was examined, and MELK mRNA
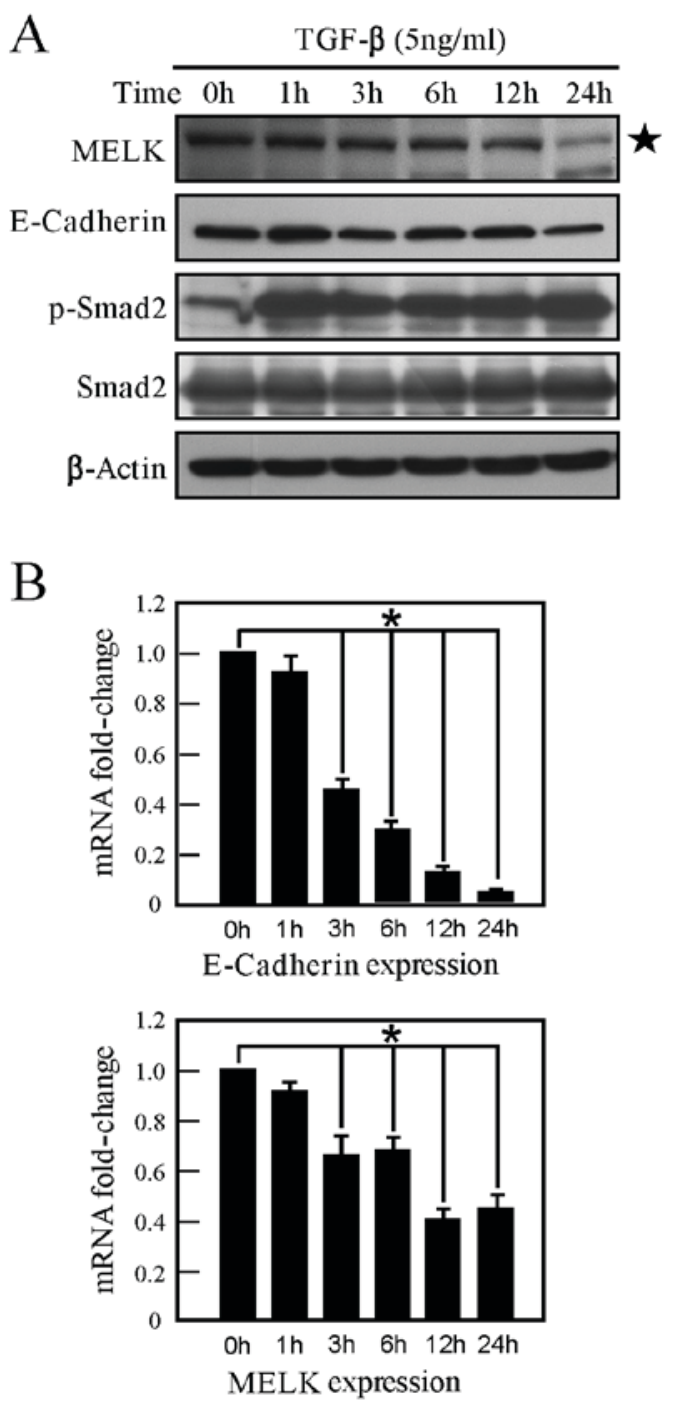

Figure 2. (A) TGF- $\beta$ inhibits MELK protein expression. The A549 cells were stimulated with TGF- $\beta 1$ for $0,1,3,6,12$ and $24 \mathrm{~h}$. MELK expression was detected by western blot analysis. The star symbol shows the MELK band. (B) TGF- $\beta$ inhibits the expression of MELK mRNA. MELK mRNA expression was detected with reverse transcription polymerase chain reaction. E-cadherin mRNA expression was detected as a positive control, demonstrating the effect of TGF- $\beta$ stimulation. The experiments were performed in triplicate. ${ }^{*} \mathrm{P}<0.05$. TGF- $\beta$, transforming growth factor- $\beta$; MELK, murine embryonic leucine zipper kinase.

expression was revealed to also be inhibited by TGF- $\beta$ (Fig. 2B). Therefore, TGF- $\beta$ can inhibit MELK expression, indicating that MELK may exhibit a number of functions in TGF- $\beta$ s signaling.

Downregulation of MELK promotes EMT. Lentivirusmediated RNA interference was used to knockdown the expression of MELK. Western blot analysis data revealed that MELK expression was decreased in MELK knockdown cells (Fig. 1A). Consistent with a previous study (18), the knockdown of MELK inhibited the proliferation of shMELK cells compared with shMock cells (Fig. 1B). shMock and shMELK cells were then treated with or without TGF- $\beta$ for $24 \mathrm{~h}$. It was identified that the E-cadherin molecules were decreased following MELK knockdown (Fig. 1C; lanes 1, 2 and 3). Following TGF- $\beta$ stimulation, it was revealed that E-cadherin 
A

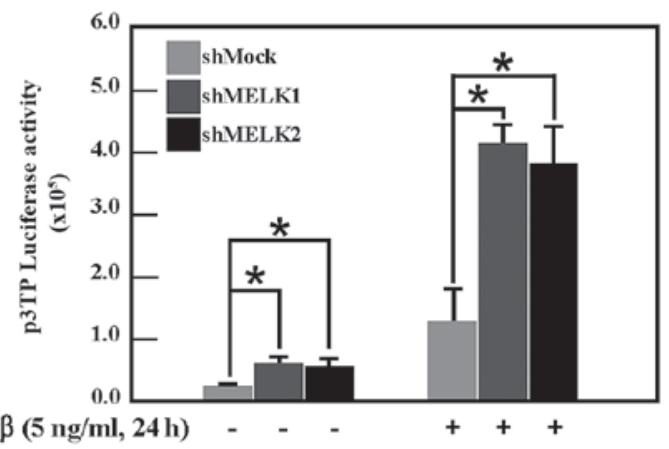

B

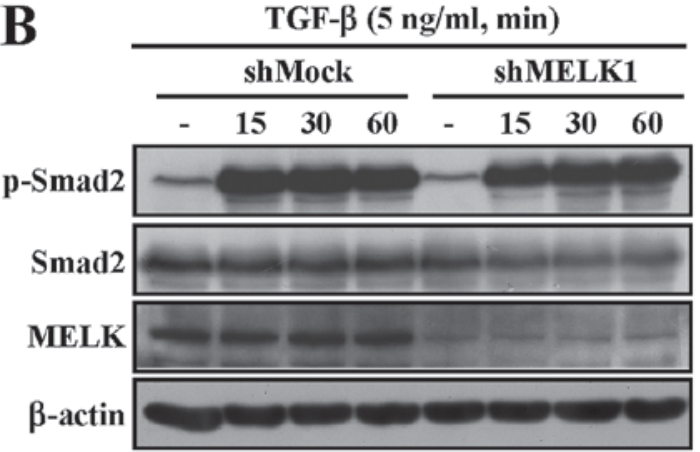

Figure 3. Knockdown of MELK enhances TGF- $\beta$ signaling activity. (A) The p3TP reporter gene was used to detect TGF- $\beta$ signaling activity in shMock cells and shMELK (sh1 and sh2) cells. The reporter gene activity was increased in shMELK cells with or without TGF- $\beta$ stimulation $\left({ }^{*} \mathrm{P}<0.05\right)$. (B) shMock and shMELK1 cells were stimulated by TGF- $\beta$ for 15,30 or 60 min, and western blot analysis was then performed using different antibodies (p-Smad2, Smad2, MELK and $\beta$-actin). The phosphorylation level of Smad 2 did not change following MELK knockdown compared with shMock cells. TGF- $\beta$, transforming growth factor- $\beta$; MELK, murine embryonic leucine zipper kinase; sh, short hairpin; p-Smad2, phosphorylated Smad2.

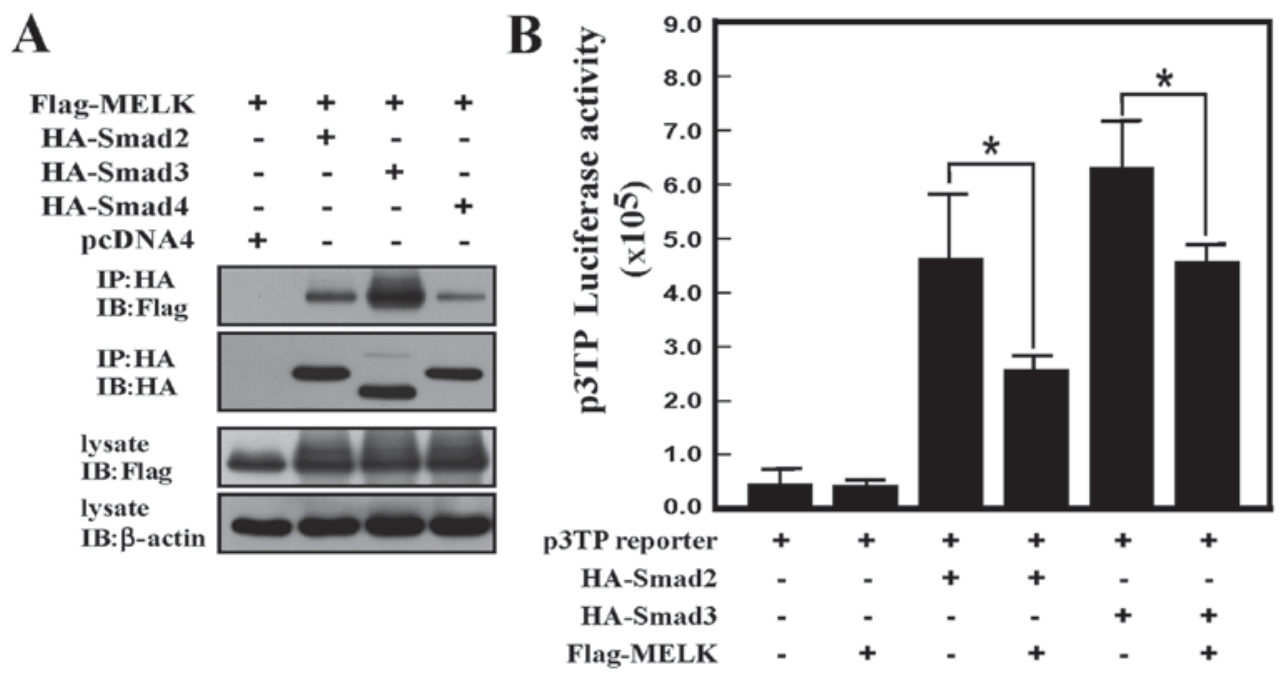

Figure 4. MELK interacts with the Smad protein and represses TGF- $\beta$ signaling activity. (A) MELK interacts with Smad2, Smad3, or Smad4 protein. The Flag-tagged MELK was co-transfected with HA-tagged Smad2, Smad3, or Smad4 plasmids. The HA antibodies were used to immunoprecipitate the immunocomplex, and the Flag antibodies were used to detect the MELK signals using western blot analysis. (B) MELK represses Smad2- or Smad3-induced activity of TGF- $\beta$ signaling. The p3TP reporter gene was transfected with different combinations of plasmids. The experiments were performed in triplicate $\left({ }^{*} \mathrm{P}<0.05\right)$. HA, hemagglutinin; TGF- $\beta$, transforming growth factor- $\beta$; MELK, murine embryonic leucine zipper kinase; IP, immunoprecipitation; IB, immnuoblot.

was further downregulated in shMock and shMELK (sh1 and sh2) cells (Fig. 1C). These results indicated that knockdown of MELK promotes EMT. Furthermore, a wound healing experiment was employed in order to observe the migration of shMock and shMELK cells. In the presence of TGF- $\beta$, shMELK (sh1 and sh2) cells were revealed to migrate faster than shMock cells (Fig. 1D). These data suggested that MELK is an important molecule in regulating EMT transition.

Knockdown of MELK enhances TGF- $\beta$ signaling activity. The marked effect of MELK on EMT suggested that MELK may regulate TGF- $\beta$ signaling activity. To test this hypothesis, a luciferase reporter gene assay was performed to analyze TGF- $\beta$ signaling in shMock and shMELK cells, using the p3TP reporter gene, which reflects the activity of TGF- $\beta$ signaling (20). Under normal culture conditions, the activity of p3TP in shMELK cells was increased compared with the activity of shMock cells (Fig. 3A). Following TGF- $\beta$ stimulation, the activity in shMELK cells was markedly increased compared with shMock cells (Fig. 3A). TGF- $\beta$ signaling was then examined by detecting the phosphorylation of Smad2 in shMock and shMELK1 cells. Data demonstrated that knockdown of MELK did not affect the phosphorylation of Smad2 (Fig. 3B). Smad 2 phosphorylation in shMELK2 cells was also examined, and no difference between shMock and shMELK2 cells was observed (data not shown). This suggested that knockdown of MELK may affect the activity of TGF- $\beta$ signaling in other ways, not through regulating the phosphorylation of Smad2 protein.

MELK interacts with Smad proteins and represses the activity of TGF- $\beta$ signaling. In order to study how MELK regulates TGF- $\beta$ signaling, it was firstly examined whether MELK can interact with Smad proteins. Using immunoprecipitation experiments, MELK was shown to interact with Smad2, Smad3 and Smad4. When MELK was used to immunoprecipitate Smad proteins, clear bands of Smad proteins were produced (Fig. 4A). The reporter gene assay was also used to 
analyze whether interaction between MELK and Smads could regulate TGF- $\beta$ signaling. As shown in Fig. 4B, overexpression of MELK can inhibit Smad2 or Smad3-induced reporter gene activity. Therefore, data from the present study revealed that the interaction between MELK and Smad proteins repressed TGF- $\beta$ signaling activity.

\section{Discussion}

Previous studies on MELK reported the function of MELK in tumorigenesis $(4,14,21)$. In the present study, the function of MELK in EMT (the initial step of metastasis) process was investigated. The results of our study identified that TGF- $\beta$ signaling can repress the expression of MELK, and that MELK can repress TGF- $\beta$ signaling through interaction with Smad proteins. In the EMT model of A549 induced using TGF- $\beta$, the knockdown of MELK promoted the cell migration (Fig. 1D).

The data from the present study, firstly disclosed that MELK is involved in TGF- $\beta$ signaling. Although knockdown of MELK did not affect the phosphorylation of Smads, it inhibited Smad2- and Smad3-induced TGF- $\beta$ activity (Fig. 4B). MELK may repress TGF- $\beta$ signaling in other ways, including the phosphorylation of other sites on Smad proteins, or affecting the location of Smad protein between the cytoplasm and nucleus.

A number of studies have already shown the marked effect of MELK inhibitors in inhibiting tumorigenesis in vivo (22-24). In these previous papers, the compound targeting MELK inhibited the proliferation of numerous cancer cell types, including the cancer stem cells $(22,24)$. In the current study, the knockdown of MELK in A549 also inhibited cell proliferation, which is concordant with previous studies. However, following the observation that MELK affects the process of EMT, the present study demonstrated that the knockdown of MELK promotes cell migration in the presence of TGF- $\beta$. In the tumor microenvironment, a number of factors, including TGF- $\beta$, are present and therefore, applying MELK inhibitor may promote the EMT and metastasis following administration of MELK inhibitors to a patient. These results require further consideration when investigating the effect of MELK inhibitor in clinical trials.

\section{Acknowledgements}

The present study was funded by the Henan Provincial Medical Science Gongguan Project (grant no. 112102310266).

\section{References}

1. Heyer BS, Warsowe J, Solter D, Knowles BB and Ackerman SL: New member of the Snf1/AMPK kinase family, Melk, is expressed in the mouse egg and preimplantation embryo. Mol Reprod Dev 47: 148-156, 1997.

2. Joshi K, Banasavadi-Siddegowda Y, Mo X, Kim SH, Mao P, Kig C, Nardini D, Sobol RW, Chow LM, Kornblum HI, et al: MELK-dependent FOXM1 phosphorylation is essential for proliferation of glioma stem cells. Stem Cells 31: 1051-1063, 2013.

3. Nakano I, Masterman-Smith M, Saigusa K, Paucar AA, Horvath S, Shoemaker L, Watanabe M, Negro A, Bajpai R, Howes A, et al: Maternal embryonic leucine zipper kinase is a key regulator of the proliferation of malignant brain tumors, including brain tumor stem cells. J Neurosci Res 86: 48-60, 2008.
4. Hebbard LW, Maurer J, Miller A, Lesperance J, Hassell J, Oshima RG and Terskikh AV: Maternal embryonic leucine zipper kinase is upregulated and required in mammary tumor-initiating cells in vivo. Cancer Res 70: 8863-8873, 2010.

5. Mirey G, Chartrain I, Froment C, Quaranta M, Bouché JP, Monsarrat B, Tassan JP and Ducommun B: CDC25B phosphorylated by $\mathrm{pEg} 3$ localizes to the centrosome and the spindle poles at mitosis. Cell Cycle 4: 806-811, 2005.

6. Jung $\mathrm{H}$, Seong $\mathrm{HA}$ and $\mathrm{Ha} \mathrm{H}$ : Murine protein serine/threonine kinase 38 activates apoptosis signal-regulating kinase 1 via Thr 838 phosphorylation. J Biol Chem 283: 34541-34553, 2008.

7. Seong HA and $\mathrm{Ha} \mathrm{H}$ : Murine protein serine-threonine kinase 38 activates p53 function through Ser15 phosphorylation. J Biol Chem 287: 20797-20810, 2012.

8. Gu C, Banasavadi-Siddegowda YK, Joshi K, Nakamura Y, Kurt H, Gupta S and Nakano I: Tumor-specific activation of the C-JUN/MELK pathway regulates glioma stem cell growth in a p53-dependent manner. Stem Cells 31: 870-881, 2013.

9. Lin ML, Park JH, Nishidate T, Nakamura Y and Katagiri T: Involvement of maternal embryonic leucine zipper kinase (MELK) in mammary carcinogenesis through interaction with Bcl-G, a pro-apoptotic member of the Bcl-2 family. Breast Cancer Res 9: R17, 2007.

10. Kuner R, Fälth M, Pressinotti NC, Brase JC, Puig SB, Metzger J, Gade S, Schäfer G, Bartsch G, Steiner E, et al: The maternal embryonic leucine zipper kinase (MELK) is upregulated in high-grade prostate cancer. J Mol Med (Berl) 91: 237-248, 2013.

11. Pickard MR, Green AR, Ellis IO, Caldas C, Hedge VL, Mourtada-Maarabouni M and Williams GT: Dysregulated expression of Fau and MELK is associated with poor prognosis in breast cancer. Breast Cancer Res 11: R60, 2009.

12. Chaffer CL and Weinberg RA: A perspective on cancer cell metastasis. Science 331: 1559-1564, 2011.

13. Xu J, Lamouille S and Derynck R: TGF-beta-induced epithelial to mesenchymal transition. Cell Res 19: 156-172, 2009.

14. Gray D, Jubb AM, Hogue D, Dowd P, Kljavin N, Yi S, Bai W, Frantz G, Zhang Z, Koeppen H, et al: Maternal embryonic leucine zipper kinase/murine protein serine-threonine kinase 38 is a promising therapeutic target for multiple cancers. Cancer Res 65: 9751-9761, 2005.

15. Moffat J, Grueneberg DA, Yang X, Kim SY, Kloepfer AM, Hinkle G, Piqani B, Eisenhaure TM, Luo B, Grenier JK, et al: A lentiviral RNAi library for human and mouse genes applied to an arrayed viral high-content screen. Cell 124: 1283-1298, 2006.

16. Chomczynski P and Sacchi N: Single Step Method of RNA Isolation by acid guanidinium thiocyanate-phenol-chloroform extraction. Anal Biochem 162: 156-159, 1987.

17. Livak KJ and Schmittgen TD: Analysis of relative gene expression data using real-time quantitative PCR and the 2(-Delta Delta C(T)) method. Methods 25: 402-408, 2001.

18. Shi J, Wang DM, Wang CM, Hu Y, Liu AH, Zhang YL, Sun B and Song JG: Insulin receptor substrate-1 suppresses transforming grow th factor-beta1-mediated epithelial-mesenchymal transition. Cancer Res 69: 7180-7187, 2009.

19. Saito RA, Watabe T, Horiguchi K, Kohyama T, Saitoh M, Nagase T and Miyazono K: Thyroid transcription factor-1 inhibits transforming growth factor-beta-mediated epithelial-to-mesenchymal transition in lung adenocarcinoma cells. Cancer Res 69: 2783-2791, 2009.

20. Datto MB, Frederick JP, Pan L, Borton AJ, Zhuang Y and Wang XF: Targeted disruption of Smad3 reveals an essential role in transforming growth factor beta-mediated signal transduction. Mol Cell Biol 19: 2495-2504, 1999.

21. Wang Y, Lee YM, Baitsch L, Huang A, Xiang Y, Tong H, Lako A, Von T, Choi C, Lim E, et al: MELK is an oncogenic kinase essential for mitotic progression in basal-like breast cancer cells. Elife 3: e01763, 2014.

22. Alachkar H, Mutonga MB, Metzeler KH, Fulton N, Malnassy G, Herold T, Spiekermann K, Bohlander SK, Hiddemann W, Matsuo Y, et al: Preclinical efficacy of maternal embryonic leucine-zipper kinase (MELK) inhibition in acute myeloid leukemia. Oncotarget 5: 12371-12382, 2014.

23. Chung S and Nakamura Y: MELK inhibitor, novel molecular targeted therapeutics for human cancer stem cells. Cell Cycle 12: 1655-1656, 2013.

24. Minata M, Gu C, Joshi K, Nakano-Okuno M, Hong C, Nguyen CH, Kornblum HI, Molla A and Nakano I: Multi-kinase inhibitor $\mathrm{C} 1$ triggers mitotic catastrophe of glioma stem cells mainly through MELK kinase inhibition. PLoS One 9: e92546, 2014. 